\title{
Microinvasive Oral Squamous Cell Carcinoma of Gingiva: An Enigma
}

\author{
Dr. Prashamsa Shakya, ${ }^{1}$ Dr. Pujan Acharya,${ }^{1}$ Dr. Shivalal Sharma, ${ }^{1}$ \\ Dr. Ashish Shrestha, ${ }^{2}$ Dr. Nisha Paunju ${ }^{3}$ \\ ${ }^{1}$ Department of Periodontology and Oral Implantology, College of Dental Surgery, \\ BP Koirala Institute of Health Sciences, Dharan, Sunsari, Nepal; \\ ${ }^{2}$ Department of Oral Pathology, College of Dental Surgery, BP Koirala Institute of Health Sciences, \\ Dharan, Sunsari, Nepal; \\ ${ }^{3}$ Department of Oral Medicine and Radiology, College of Dental Surgery, BP Koirala Institute of Health Sciences, \\ Dharan, Sunsari, Nepal.
}

\begin{abstract}
Microinvasive oral squamous cell carcinoma (MIOSCC) of gingiva is an early stage relatively 'thin' tumour without invasion of deep tissues. A 55-year-old male reported with the chief complaint of gum swelling in lower right back jaw region for one month. On examination, diffuse, unscrappable, white homogenous plaque extending from 35 to 48 was present with a firm, sessile gingival growth approximately eight millimetre in diameter. A provisional diagnosis of verrucous leukoplakia was made. Hence, biopsy was performed which confirmed MIOSCC and mild dysplasia for different sites. The patient is on regular follow-up and with no signs of recurrence.

Keywords: Biopsy; microinvasive oral squamous cell carcinoma; verrucous leukoplakia.
\end{abstract}

\section{INTRODUCTION}

Squamous cell carcinoma is the most common malignant neoplasm of the oral cavity and represents about $90 \%$ of all oral malignancies. ${ }^{1}$ One aspect of squamous cell carcinoma that requires consideration is microinvasive squamous cell carcinoma which is an early stage relatively 'thin' tumour without invasion of deep tissues. ${ }^{2}$

American Joint Committee on Cancer (AJCC) and Union for International Cancer Control (UICC) define "microinvasive carcinoma" as a lesion that is predominantly intraepithelial with a focus of invasion of microscopic dimensions confined to superficial stroma or lamina propria. ${ }^{3}$

This lesion is challenging concerning its clinical presentation, metastatic ability, therapeutic intervention, and prognosis. ${ }^{4}$

\section{CASE REPORT}

A 55-year-old medically fit male reported to the Department of Periodontology and Oral Implantology with the chief complaint of outgrowth of mass in lower right back teeth

Correspondence
Dr.Prashamsa Shakya
Email: prashamsa86@gmail.com
Citation
ShakyaP, AcharyaP, SharmaS, Shrestha A, Paunju N. Microinvasive
Oral Squamous Cell Carcinoma of Gingiva: An Enigma. J Nepal Soc
Perio Oral Implantol. 2021 Jan-Jun;5(9):57-60.

region for one month which was progressively increasing in size and not associated with pain or bleeding. He had a habit of chewing tobacco for 20 years but had quit recently after the appearance of the lesion.

On extraoral examination, no abnormalities were detected. Intraoral examination revealed a firm, sessile, proliferative gingival growth of around $8 \mathrm{~mm} \times 8 \mathrm{~mm}$ in respect to 44 (according to two-digit numbering system). Diffuse, unscrappable, white homogenous plaque was present on the buccal mucosa, vestibule, and gingiva extending from 35 to 48 region (Figure 1). Radiographically, no abnormalities were detected (Figure 2).

The laboratory tests were within normal limits. A provisional diagnosis of verrucous leukoplakia was made.

In phase I therapy, scaling and root planing (SRP) was done. An incisional biopsy was performed in relation to 34,35 , and 36 (Figure 3) and punch biopsy (Figure 4) was done in right buccal mucosa under local anaesthesia (Figure 5,6). The specimen was then sent to the Department

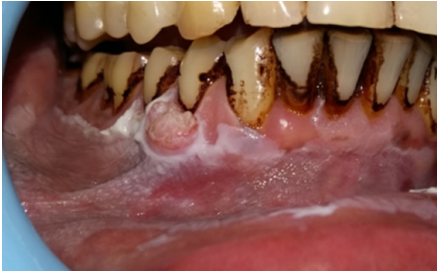

Figure 1: Preoperative view.

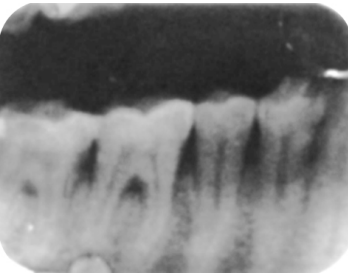

Figure 2: Intraoral periapical radiograph.

This is an open access journal, and articles are distributed under the terms of the Creative Commons Attribution CC BY 4.0 Licence. 


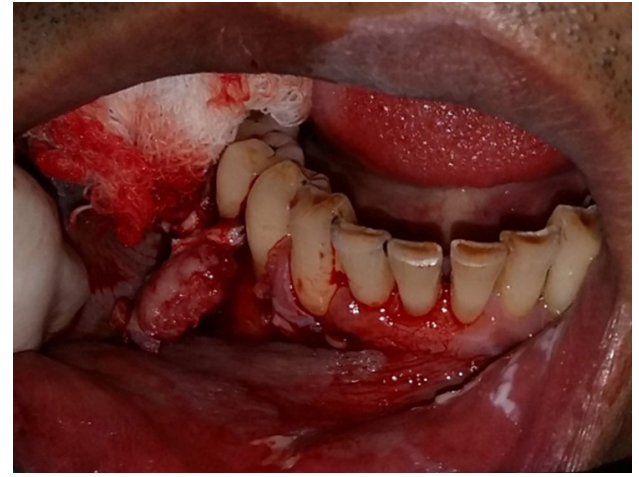

Figure 3: Excision of mass.

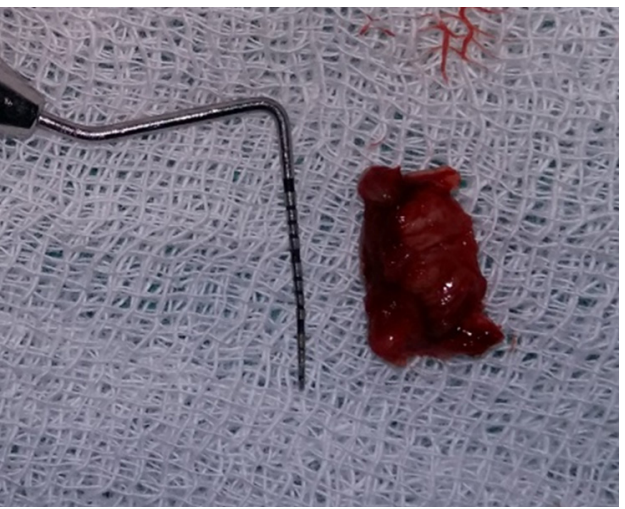

Figure 5: Excised mass size compared with UNC-15

probe.

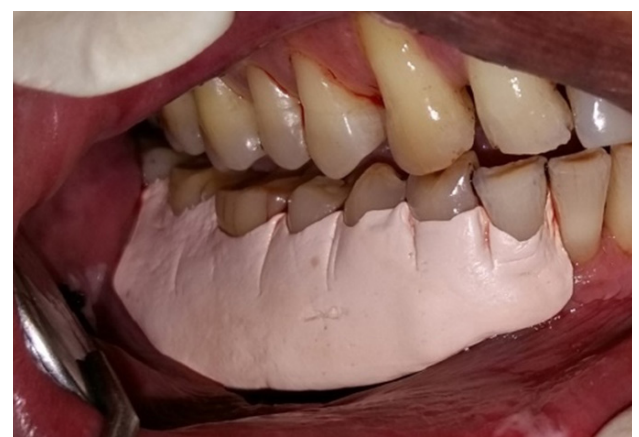

Figure 7: Periodontal dressing (Coe-pak) placed.

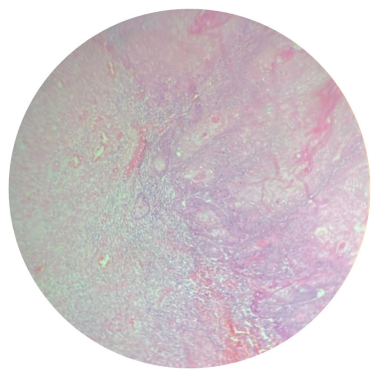

Figure 9: Histopathology at 10X magnification.

of Oral Pathology for histopathological examination. Periodontal dressing (Figure 7) was applied locally at the surgical site in gingiva and sutures were placed at buccal mucosa (Figure 8). Ibuprofen $400 \mathrm{mg}$ thrice daily for two days was prescribed for post-operative pain management. Chlorhexidine mouthwash $0.2 \%$ twice-daily for two weeks was prescribed. Oral hygiene instructions were given.

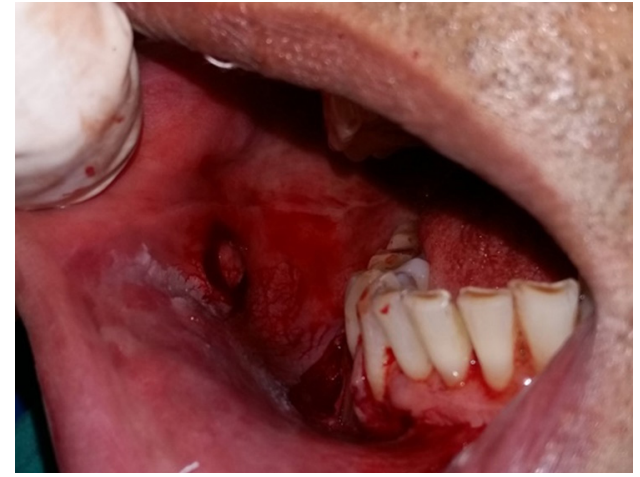

Figure 4: Punch biopsy.

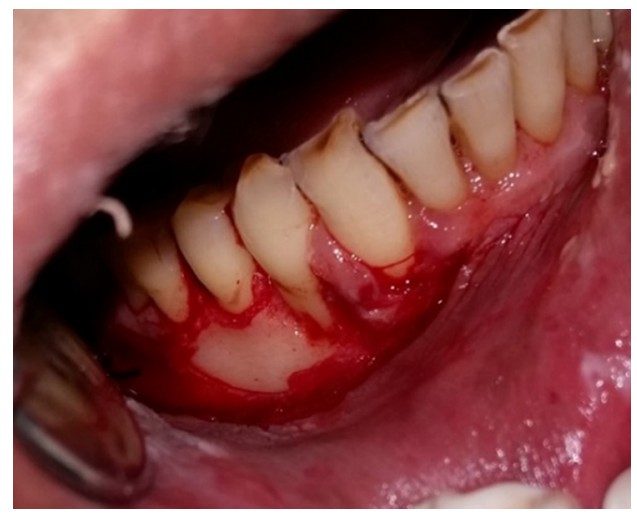

Figure 6: Post-operative view.

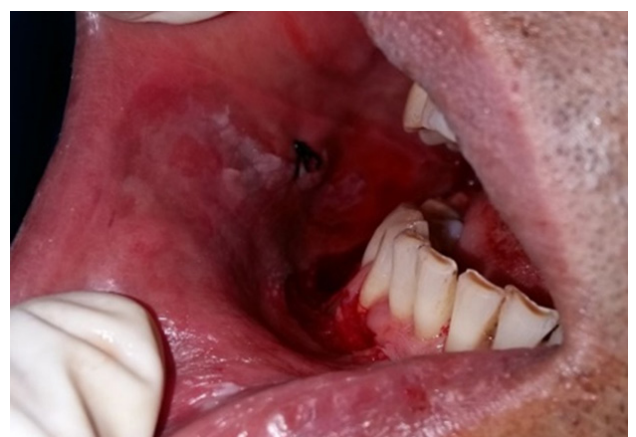

Figure 8: Sutures placed.

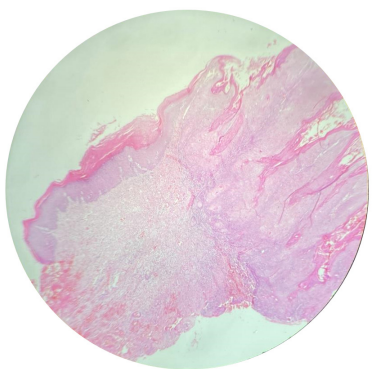

Figure 10: Histopathology at 4X magnification.

Histopathological section under $4 \mathrm{X}$ magnification revealed invasive tumour front along with multiple epithelial tumour islands present within lamina propria (Figure 9). Histopathological section under 10X magnification revealed islands with dysplastic features in the form of cellular and nuclear pleomorphism, nuclear hyperchromatism, and mitotic activities (Figure 10). 


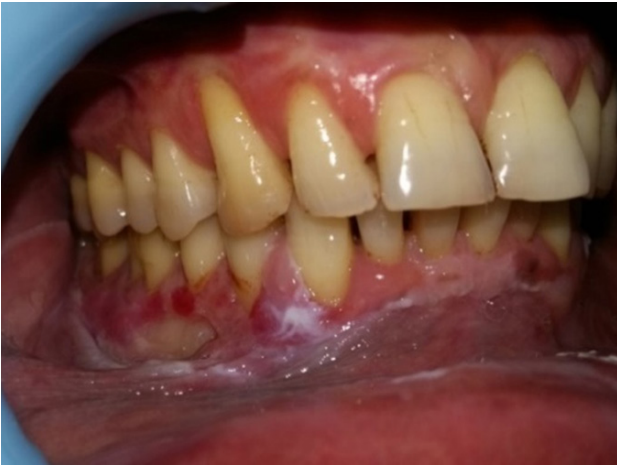

Figure 11: Healing at seven days.

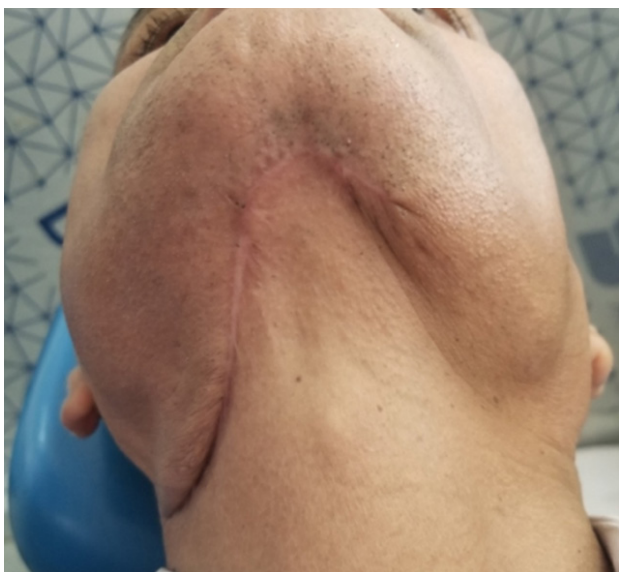

Figure 13: Submental scar.

These findings were suggestive of "microinvasive oral squamous cell carcinoma (MIOSCC)."

Contrast Enhanced Computed Tomography (CECT) of the neck and mandible revealed mild thickening and enhancement of right mandibular gingiva in relation to 34,35 , and 36 along with mild mucosal wall thickening of the adjacent inner buccal cavity with no evidence of adjacent muscular wall infiltration or bony erosion/remodeling.

The healing at seven-day and one-month follow-up has been depicted in Figure 11 and Figure 12 respectively. The patient then approached B.P. Koirala memorial cancer hospital, Bharatpur, Chitwan for further treatment where he underwent wide local excision with marginal mandibulectomy right side with right supra-omohyoid neck dissection and reconstruction with submental island flap.

Resected mandible sent for histopathological examination also revealed MIOSCC of right gingiva with five mm thickness and two mm depth of invasion (pT1NOMX).

The patient revisited us four months after recovery from surgery. No evidence of recurrence was noted. The patient is under regular follow-up and has been planned for prosthodontic rehabilitation.

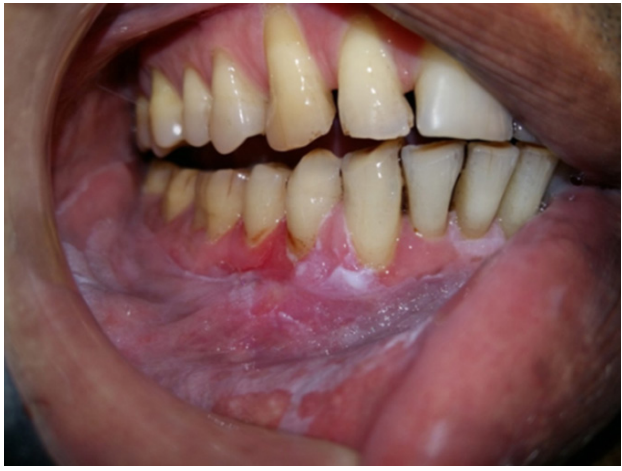

Figure 12: Healing at one month.

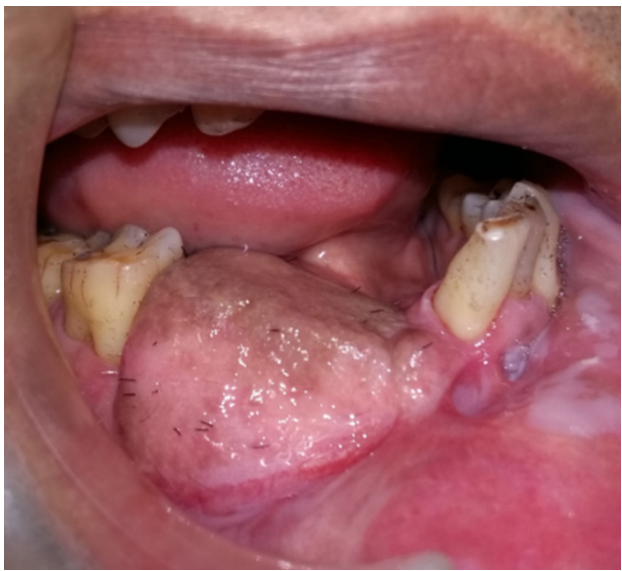

Figure 14: Follow-up at 15 months.

\section{DISCUSSION}

There is extensive literature related to oral cancer, particularly with respect to the cause, pathogenesis, and treatment of oral squamous cell carcinoma (SCC). Despite abundant data related to overall SCC of the mouth, relatively little is known concerning MIOSCC of the gingiva specifically.

Pentenero et al. ${ }^{3}$ found that MIOSCC were not significantly related to gender, age, tobacco or alcohol habit, presence of symptoms, mucosal type, site, or location of the lesions. It had distinctive clinical features presenting more frequently as patches, plaques, or erosions rather than as ulcers or verrucous lesions and were an incidental finding during routine dental visits or during follow-up visits for premalignant disorders. In the present case, the patient had the habit of placing tobacco in the associated region and presented with plaques as well as verrucous growth in the gingiva.

MIOSCC generally presents itself clinically as one of the oral potentially malignant disorders thus a high degree of caution is essential to prevent misdiagnosis of such lesions. The depth of invasion of MIOSCC is very less, generally between 0.5 to two mm. Microinvasion is purely a histopathological 
diagnosis, the hallmark being the breach in basement membrane along with the presence of dense inflammatory cell infiltrate. ${ }^{5}$

A classification system for MIOSCC is difficult and has yet not been established owing to the limited literature. In the absence of a universally accepted treatment protocol for MIOSCC with clinically or cytopathological negative nodes, surgical treatment aimed at excising the lesions with one to two mm margins at the periphery and deep margins is recommended for these lesions. ${ }^{6}$ Elective neck dissection is still a controversy and is debatable. A thorough examination of the lymphatic drainage of the affected area may be warranted. ${ }^{2}$

Regmee et al. ${ }^{7}$ reported an erythematous erosive lesion in gingiva mimicking oral lichen planus where local excision of the lesion was done. However, wide local excision with marginal mandibulectomy with right supra-omohyoid neck dissection and reconstruction with submental island flap was done in this case.

Although the number of well-documented cases of MIOSCC is small, it is thought that the post-operative prognosis of MIOSCC is good and that distant metastases are rare. Even though oral cancers are a relatively rare occurrence in the realm of the periodontium, this case demonstrates that carcinoma can mimic oral potentially malignant disorders and serves as a reminder to keep the possibility of carcinoma in mind when examining intraoral lesions. Emphasis is placed upon histopathologic examinations to arrive at a definitive diagnosis.

Conflict of Interest: None.

\section{REFERENCES}

1. Johnson NW, Jayasekara P, Amarasinghe AA. Squamous cell carcinoma and precursor lesions of the oral cavity: Epidemiology and aetiology. Periodontol 2000. 2011 Oct;57(1):19-37.

2. Shankar AA, Gokul S. The dilemma of microinvasion. Head Neck Oncol. 2014 Jul 18;6(3):30-1.

3. Pentenero M, Navone R, Motta F, Marino R, Gassino L, Broccoletti R, Gandolfo S. Clinical features of microinvasive stage I oral carcinoma. Oral Dis. 2011 Apr; 17(3):298-303.

4. Byatnal AA, Natarajan J, Shenoy S, Kamath A, Hunter K, Radhakrishnan R. A 3-dimensional assessment of the depth of tumour invasion in microinvasive tongue squamous cell carcinoma - A case series analysis. Med Oral Patol Oral Cir Bucal. 2015 Nov 1;20(6):e645-50.

5. Bianchi S, Vezzosi V. Microinvasive carcinoma of the breast. Pathol Oncol Res. 2008;12:105-11.

6. Carroll WR. Surgery for cancer of the oral cavity. In: Fisher JE, Bland KI. Master of surgery. 5th ed. Lippincott Williams and Wilkins. 2007;1:308

7. Regmee P, Rimal J, Maharjan IK, Shrestha A, Niroula D, Luitel A, et al. Microinvasion: A clinical dilemma. Kathmandu Univ Med J. 2019;65(1):72-4. 